\title{
Nutrition assessment - Modal ergometry
}

Avaliação NUTROLógICA - ERGoMETRIA MOdAL

Authorship: Associação Brasileira de Nutrologia (ABRAN)

Participants: Ribas $\mathrm{DF}^{1}$, Kelman $\mathrm{G}^{2}$, Buzzini $\mathrm{RF}^{2}$, Simões RS ${ }^{2}$, Bernardo $\mathrm{WM}^{2}$

Final draft: March 11, 2016

${ }^{1}$ Sociedade Brasileira de Nutrologia

${ }^{2}$ Programa Diretrizes, Brazilian Medical Association

The Guidelines Project, an initiative of the Brazilian Medical Association, aims to combine information from the medical field in order to standardize procedures to assist the reasoning and decision-making of doctors.

The information provided through this project must be assessed and criticized by the physician responsible for the conduct that will be adopted, depending on the conditions and the clinical status of each patient.

\section{EVIDENCE COLLECTION METHOD}

This policy followed the pattern of a systematic review with retrieval of evidence based on the principles of evidence-based medicine (EBM), according to which clinical experience is integrated with the ability to critically analyze and rationally apply scientific information, thus improving the quality of medical care. EBM uses existing scientific evidence available at the time, with good internal and external validity, applying its results to the clinical practice. ${ }^{1,2}$

Systematic reviews are considered today as level I evidence for any clinical question as systematically summarize information on a particular topic based on primary studies (clinical trials, cohort studies, case-control or cross-sectional studies). The methodology used for this is reproducible, and integrates information on effectiveness, efficiency, efficacy, and safety. ${ }^{1,2}$

We ask the questions in a structured way, summarized by the acronym PICO, where $\mathrm{P}$ is the patient or population, I intervention or indicator, $\mathrm{C}$ is comparison or control, and $\mathrm{O}$ is the outcome. Based on a structured question, the keywords or descriptors that will form the basis of the search for evidence in the various available databases are identified ${ }^{1,2}$ (Annex I).

\section{Clinical question}

What is the role of modal ergometry in the measurement of muscle strength in children, adolescents or adults?

\section{Grade of Recommendation AND StREnGth OF EVIDENCE}

- A: Experimental or observational studies of higher consistency.

- B: Experimental or observational studies of lower consistency.
- C: Case reports/non-controlled studies.

- D: Opinions without critical evaluation, based on consensus, physiological studies or animal models.

\section{Objective}

To determine the role of modal ergometry in the measurement of muscle strength while assessing the nutrition status of children, adolescents and adults.

\section{Conflict Of InTERest}

No conflict of interest was declared by the participants in the development of this guideline.

\section{INTRODUCTION}

Impairment of muscle strength is a well-known phenomenon that occurs in diseases related to poor nutrition. A reduced nutritional supply results in compensatory loss of body protein, mostly from the muscles. Decline in muscle protein synthesis can also occur in diseases related to poor nutrition. Reduced muscle strength is, in turn, associated with a loss of physical functionality and a negative impact on the recovery of acute diseases or surgery, which partly explains a high predictive power of muscle function tests. The palmar gripping force test reflects the maximum strength derived from the contraction of intrinsic and extrinsic muscles that support the contraction of the joints in the hands. Despite its good correlation with other muscle function tests, such as the knee extension test and the peak expiratory flow test, it cannot be used to replace the evaluation of lower-limb muscle function. ${ }^{3}(\mathbf{D})$

Approximately 9 to $35 \%$ of patients admitted with a diagnosis of acute stroke, either ischemic or hemorrhagic, are malnourished. Dysphagia contributes to poor di- 
etary intake and is present in over $50 \%$ of patients with acute stroke, further increasing the risk of malnutrition in these patients. Neurological, emotional, and cognitive changes can also affect the nutrition of these patients. Elderly patients $(\mathrm{n}=170)$ with diagnosis of stroke who presented malnutrition or nutritional risk [body mass index $(\mathrm{BMI}) \leq 20 \mathrm{~kg} / \mathrm{m}^{2}$, or unintentional loss of weight greater than $5 \%$ in the last 3 to 6 months, or decreased dietary intake in the last 5 days] were randomized to receive personalized nutrition combined with supplementation or routine care (control). Weight loss after 3 months of follow-up was higher in the control group, but the difference was not statistically significant. There was a significant increase in palmar gripping force in the intervention group, and decreased strength in the control group $(73.2$ vs. $44.6 \%, \mathrm{p}=0.001) .{ }^{4}(\mathbf{B})$

In cancer patients, malnutrition is common and is associated with worse quality of life, increased morbidity and mortality. One hundred and thirty patients aged 19 to 95 years, with malnutrition $(22.3 \%)$ or risk of malnutrition (42.3\%), were followed during hospitalization in a cancer hospital. One hundred and eleven (111) patients had solid tumors and 19 hematopoietic neoplasms. Patients with less palmar gripping force on admission, which accounted for more than half of the total, were discharged later (> 15 days of hospitalization), while those with higher values for strength stayed in the hospital for a shorter period (discharged earlier than 15 days). Patients malnourished or at risk of malnutrition and those with low palmar gripping force values on admission were associated with increased risk of death during hospitalization, regardless of age. This allowed us to distinguish who would have a long hospital stay (Table 1). ${ }^{5}$ (B)

\section{TABLE 1 Palmar gripping force.}

\begin{tabular}{lll}
$\begin{array}{l}\text { Palmar gripping } \\
\text { force }\end{array}$ & $\begin{array}{l}\text { Length of hospital } \\
\text { stay-IQR }\end{array}$ & $\begin{array}{l}\text { p-value hospital } \\
\text { discharge }\end{array}$ \\
\hline High & $6(4.0-11.0)$ & $<0.001$ \\
\hline Intermediate & $12(7.3-23.3)$ & $<0.001$ \\
\hline Low & $17(7.0-32.0)$ & $<0.001$ \\
\hline IQR: interquartile range. & &
\end{tabular}

In order to assess palmar gripping force as a screening method for the identification of patients (18 to 96 years old) considered malnourished in hospital environment, 314 individuals with diseases including cardiovascular, gastrointestinal, respiratory, neurologic, surgical, and more were evaluated. All patients underwent the palmar gripping force test, performed using a mechanical dyna- mometer. The Nutritional Risk Screening (NRS-2002) protocol was applied to assess the nutritional risk.

Patients identified as malnourished according to the NRS-2002 (37.9\%) had low values of palmar gripping force $(\mathrm{p}<0.001)$. When patients with decreased palmar gripping force were compared with those with greater force, this parameter showed good specificity (70.2\%) and sensitivity $(86.7 \%)$, and positive and negative predictive values, respectively, 69.9 and $86.8 \%$, with $77.4 \%$ agreement, $\mathrm{k}=0.56$. Thus, it proved to be an appropriate tool for nutritional screening in hospitals. ${ }^{6}(\mathbf{B})$

The liver is an essential metabolic organ for energy-protein control in the body. Patients with liver disease show a greatly affected nutritional status, and energy-protein malnutrition is found in most patients with cirrhosis. Prevalence of ascites, postoperative mortality, and post-transplant prognosis are all related to energy-protein malnutrition. One hundred and forty-five (145) patients were followed up; they were divided into three groups (group 1 with 50 patients with cirrhosis; group 2, 46 patients with hypertension; and group 3, 49 patients with functional gastrointestinal disease, which is the control group). According to the gold standard Subjective Global Assessment (SGA), malnutrition was found in $28 \%$ of patients in group $1,13.1 \%$ in group 2 , and there were no malnourished patients in group 3 . The palmar gripping force test led to the detection of $63 \%$ of the malnourished patients in group $1,12.7 \%$ in group 2 , and $4.08 \%$ in group 3. The sensitivity of the palmar gripping force test was $100 \%$, while specificity reached $48.6 \%$, positive predictive value amounted to $37.9 \%$, and negative value to $100 \%$; $\mathrm{k}$ test $=0.31$ compared with the gold standard $(\mathrm{SGA}) .^{7}(\mathbf{B})$

Assessing the palmar gripping force of 787 healthy children from 6 to 10 years old, we identified a difference in mean gripping force between the malnourished and the well-nourished children, which was more noticeable among the older children. Of the 116 boys and 112 girls classified as malnourished using weight for age, only 24 and $22.3 \%$, respectively, had low values for grip strength. ${ }^{8}(\mathbf{B})$

When grip strength and height for age were used to assess the prevalence of malnutrition, only $26.7 \%$ of the boys and $22.9 \%$ of the girls with short stature presented low values for gripping force. Similar results were observed in children considered malnourished based on height for weight. ${ }^{8}(\mathbf{B})$

Sensitivity of the muscle strength test as a nutritional status index was not as high for both sexes, and 39.3\% was the highest value when the strength test was compared with the height for age among the girls. Specificity of the strength test was $94 \%$ compared with weight for age, and 
94.3\% when compared to height for age in boys. The positive predictive value of the strength test compared with the two methods was $70 \%$. However, in the case of weight for height, it reached low values $(25.5 \%$ for girls and $35.5 \%$ for boys) (Table 2$).{ }^{8}(\mathbf{B})$

TABLE 2 Sensitivity, specificity, and PPV of the muscle strength test for both sexes. ${ }^{8}$ (B)

\begin{tabular}{lllllll} 
& \multicolumn{3}{l}{ Weight/Age } & \multicolumn{2}{l}{ Height/Age } & \multicolumn{2}{l}{ Weight/Height } \\
\hline & M & F & M & F & M & F \\
\hline Sensitivity (\%) & 24.7 & 27.3 & 26.7 & 39.3 & 23.9 & 25.9 \\
\hline Specificity (\%) & 94.0 & 87.8 & 94.3 & 76.5 & 87.4 & 85.1 \\
\hline PPV & 70.0 & 43.9 & 70 & 22.9 & 31.5 & 21.5
\end{tabular}

PPV: positive predictive value; $\mathrm{M}$ : male; $\mathrm{F}$ : female.

\section{Recommendations}

The palmar gripping force test is an appropriate tool for nutritional assessment and screening of adult and elderly individuals hospitalized due to cardiovascular, gastrointestinal, respiratory, neurologic, surgical, and other diseases. The test also allows the estimation of hospital stay and risk of death during hospitalization of patients with cancer. In patients with liver cirrhosis, the palmar gripping force test is a good method to predict increased incidence of major complications. In children, the palmar gripping force test proved to be specific to measure lean body mass; however, sensitivity was low when compared to weight for age, height for age, and weight for height, which suggests that only children with decreased lean mass could be identified as malnourished.

\section{References}

1. Nobre MR, Bernardo WM, Jatene FB. A prática clínica baseada em evidências. Parte I - Questões clínicas bem construídas. Rev Assoc Med Bras. 2003; 49(4):445-9.

2. Bernardo WM, Nobre MR, Jatene FB. A prática clínica baseada em evidências. Parte II - Buscando as evidências em fontes de informação. Rev Assoc Med Bras. 2004; 50(1):104-8.

3. Norman K, Stobäus N, Gonzalez MC, Schulzke JD, Pirlich M. Hand grip strength: outcome predictor and marker of nutritional status. Clin Nutr. 2011;30(2):135-42.

4. Ha L, Hauge T, Spenning AB, Iversen PO. Individual, nutritional support prevents undernutrition, increases muscle strength and improves QoL among elderly at nutritional risk hospitalized for acute stroke: a randomized, controlled trial. Clin Nutr. 2010; 29(5):567-73.

5. Mendes J, Alves P, Amaral TF. Comparison of nutritional status assessment parameters in predicting length of hospital stay in cancer patients. Clin Nutr. 2014; 33(3):466-70.

6. Matos LC, Tavares MM, Amaral TF. Handgrip strength as a hospital admission nutritional risk screening method. Eur J Clin Nutr. 2007; 61(9):1128-35

7. Alvares-da-Silva MR, Reverbel da Silveira T. Comparison between handgrip strength, subjective global assessment, and prognostic nutritional index inassessing malnutrition and predicting clinical outcome in cirrhotic outpatients. Nutrition. 2005; 21(2):113-7.

8. Kenjle K, Limaye S, Ghugre PS, Udipi SA. Grip strength as an index for assessment of nutritional status of children aged 6-10 years. J Nutr Sci Vitaminol (Tokyo). 2005; 51(2):87-92. 


\section{Annex I}

\section{Clinical question}

What is the role of modal ergometry in the measurement of muscle strength in children, adolescents or adults?

\section{Structured QUeStion}

- P: Children, adolescents or adults

- I: Modal ergometry

- $\mathrm{C}:$

- O: Measurement of muscle strength

\section{Strategy FOR SEARCH OF EVIDENCE}

- \#1 - (Nutrition Disorder OR Nutritional Disorders OR Nutritional Disorder OR Nutritional Status OR Nutrition Status OR Nutrition Assessment OR Nutrition Disorders OR Malnutrition OR Deficiency Diseases OR Overnutrition OR Obesity OR Avitaminosis OR Ascorbic Acid Deficiency OR Vitamin A Deficiency OR Vitamin B Deficiency OR Vitamin D Deficiency OR Vitamin E Deficiency OR Vitamin K Deficiency OR Magnesium Deficiency OR Potassium Deficiency OR Protein Deficiency OR Protein-Energy Malnutrition OR Swayback OR Scurvy OR Choline Deficiency OR Folic Acid Deficiency OR Hyperhomocysteinemia OR Pellagra OR Riboflavin Deficiency OR Thiamine Deficiency OR Beriberi OR Wernicke Encephalopathy OR Vitamin B 12 Deficiency OR Anemia, Pernicious OR Subacute Combined OR Degeneration OR Vitamin B 6 Deficiency OR Rickets OR Osteomalacia OR Renal Osteodystrophy OR Steatitis OR Kwashiorkor OR Overweight OR Obesity, Abdominal OR Obesity, Morbid OR Wasting Syndrome) $=753,330$

- \#2 - (Hand Strength OR Muscle Strength OR Muscle Weakness OR Ergometry OR Ergometer OR Modal Ergometry OR dynamometer) $=146,656$

\section{Modal ergometry $=\# 1$ AND \#2 = \#3=6,750}

Methodological search filter $=\# 4=(($ specificity $[$ Title $)$ Abstract]) OR random* OR ((prognos*[Title/Abstract] OR (first[Title/Abstract] AND episode[Title/Abstract]) OR cohort[Title/Abstract]))) $=2,045,824$

$1^{\text {st }}$ Retrieval $=\# 3$ AND \#4 $=1,434$

- (((Nutrition Disorder OR Nutritional Disorders OR Nutritional Disorder OR Nutritional Status OR Nu- trition Status OR Nutrition Assessment OR Nutrition Disorders OR Malnutrition OR Deficiency Diseases OR Overnutrition OR Obesity OR Avitaminosis OR Ascorbic Acid Deficiency OR Vitamin A Deficiency OR Vitamin B Deficiency OR Vitamin D Deficiency OR Vitamin E Deficiency OR Vitamin K Deficiency OR Magnesium Deficiency OR Potassium Deficiency OR Protein Deficiency OR Protein-Energy Malnutrition OR Swayback OR Scurvy OR Choline Deficiency OR Folic Acid Deficiency OR Hyperhomocysteinemia OR Pellagra OR Riboflavin Deficiency OR Thiamine Deficiency OR Beriberi OR Wernicke Encephalopathy OR Vitamin B 12 Deficiency OR Anemia, Pernicious OR Subacute Combined OR Degeneration OR Vitamin B 6 Deficiency OR Rickets OR Osteomalacia OR Renal Osteodystrophy OR Steatitis OR Kwashiorkor OR Overweight OR Obesity, Abdominal OR Obesity, Morbid OR Wasting Syndrome) AND (Hand Strength OR Muscle Strength OR Muscle Weakness OR Ergometry OR Ergometer OR Modal Ergometry) AND ((diagnosis/broad [filter]) OR random* OR ((prognos*[Title/Abstract] OR (first[Title/ Abstract] AND episode[Title/Abstract]) OR cohort[Title/ Abstract $])))))$ NOT $((((($ ergometry OR ergometer OR model ergometry) AND (strength OR muscle OR weakness) AND (diagnosis/broad [filter] OR prognosis/ broad [filter] OR random*))) OR ((hand strength OR muscle strength OR muscle weakness) AND (ergometry OR ergometer OR modal ergometry) and ((diagnosis/broad [filter]) or random* or ((prognos*[title/ abstract] or (first[title/abstract] and episode[title/abstract]) or cohort[title/abstract]))))))

\section{Studies Retrieved}

The number of studies retrieved until the last day of searching $(03 / 11 / 16)$ according to the final search strategy was 3,292 .

\section{EXCLUSION CRITERIA}

Articles that did not meet the specificities of PICO, that were not available for access in full, and those written in languages other than English, Portuguese or Spanish were excluded. 